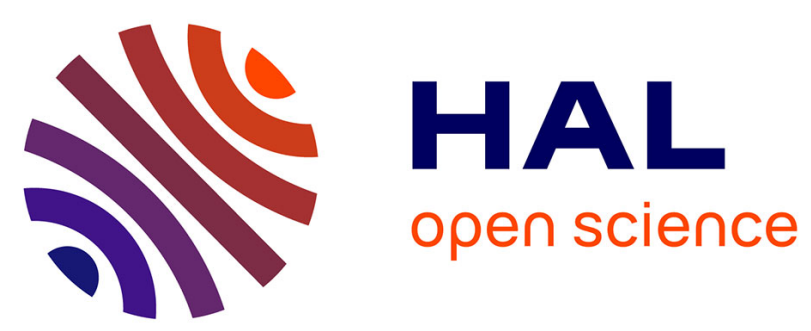

\title{
Angkor à l'âge du multimedia
}

Christophe Pottier

\section{To cite this version:}

Christophe Pottier. Angkor à l'âge du multimedia. Aséanie, Sciences humaines en Asie du Sud-Est, 1998, 2 (1), pp.213-222. 10.3406/asean.1998.1612 . halshs-02043449

\section{HAL Id: halshs-02043449 \\ https://shs.hal.science/halshs-02043449}

Submitted on 21 Feb 2019

HAL is a multi-disciplinary open access archive for the deposit and dissemination of scientific research documents, whether they are published or not. The documents may come from teaching and research institutions in France or abroad, or from public or private research centers.
L'archive ouverte pluridisciplinaire HAL, est destinée au dépôt et à la diffusion de documents scientifiques de niveau recherche, publiés ou non, émanant des établissements d'enseignement et de recherche français ou étrangers, des laboratoires publics ou privés. 


\section{Angkor à l'âge du multimedia}

In: Aséanie 2, 1998. pp. 213-222.

Citer ce document / Cite this document :

Pottier Christophe. Angkor à l'âge du multimedia. In: Aséanie 2, 1998. pp. 213-222.

doi : 10.3406/asean.1998.1612

http://www.persee.fr/web/revues/home/prescript/article/asean_0859-9009_1998_num_2_1_1612 


\section{ANGKOR À L’ÂGE DU MULTIMÉDIA}

\section{Angkor, Cité royale [Récit de voyages]}

Réalisation: Julien FrYDMAN, 1997

CD-Rom MAC/PC

Édition: Infogrames Multimédia

Coproduction: Réunion des Musées Nationaux - UNESCO - Infogrames

Production exécutive et développement: Le Lab

Villeurbanne

\section{Angkor, 10 siècles de fascination}

Réalisation:

Fabrice CEREZAles et Jean-François CoulaIS, 1997

CD-Rom MAC/PC

Avec la collaboration scientifique de Jacques Dumarçay, Bruno Dagens

et Fabienne Luco

Coproduction: Comme un Voyage - Studio Cerezales - nLoops - AFAA IDEA production - Polka.

Préface de S.A.R. Norodom Sihanouk, roi du Cambodge

Photographie et vidéo: Bruno Thommassin

Conception graphique et interactivité: Frédéric Locca

Paris

Comme toutes les grandes expositions culturelles parisiennes, l'exposition "Angkor et dix siècles d'Art khmer", qui s'est tenue aux Galeries nationales du Grand Palais à Paris, du 31 janvier au 26 mai 1997, a été accompagnée par une vague de publications, allant de l'imposant catalogue officiel de l'exposition au numéro spécial de magazines et, depuis peu, le cédérom. Après leur premier tour du Monde, les 117 pièces et statues ont retrouvé il y a peu leur place dans leurs musées respectifs. Les laissant reposer, on se permettra ici un petit retour en arrière sur les deux cédéroms qui sont parus à Paris à l'occasion de cette exposition. 
Le premier cédérom était sorti à temps pour profiter pleinement de la déferlante de l'exposition: Angkor, Cité royale [Récit de voyages] réalisé par Julien Frydman, coproduit par la Réunion des Musées Nationaux, l'UNESCO et Infogrames. Bien placé sur les présentoirs, il s'agit donc du cédérom officiel. Claude Jacques et Thierry Zéphir apparaissent sur la jaquette pour la "validation scientifique" (respectivement pour l'UNESCO et la RMN).

Le second cédérom était en retard de quelques semaines et sa distribution ne profita donc que partiellement de la promotion des premiers jours de l'exposition: Angkor, 10 siècles de fascination, réalisé par Fabrice Cerezales avec la collaboration scientifique de Jacques Dumarçay, Bruno Dagens et Fabienne Luco, coproduit par Comme un Voyage, Studio Cerezales, nLoops, AFAA, IDEA production et Polka. Un cédérom non-officiel, que l'on n'oserait pas cependant qualifier de pirate avec sa préface de S.A.R. Norodom Sihanouk et (entre autres) le sigle de l'EFEO au dos de la boîte.

Autour du même événement, la concurrence était inévitable et la comparaison a priori tentante. Pourtant ces deux cédéroms de format $\mathrm{MAC} /$ PC ont beau avoir un titre voisin, de nombreux points les séparent. Ils nous offrent ainsi deux "produits" distincts, voire opposés, sur un même support. Voyons-les donc séparément.

\section{Angkor, Cité royale [Récit de voyages]}

L'introduction de ce cédérom présente le récit d'un jeune touriste mélancolique qui s'envole visiter Angkor en solitaire: un séjour de 7 jours, Phnom Penh compris, sur les traces de son grand-père qui aurait " vécu làbas dans les années 30". Le ton nostalgique du scénario est donné, avec ambiance de vieux films en noir et blanc, de danseuses du début du siècle sur fond sépia et une carte où s'inscrivent les sauts de puce de l'avion de ce grand-père. Doit-on alors voir ces escales aux noms évocateurs d'aventure comme une référence (au énième degré) au trajet d'un Indiana Jones IV? Et la bordure de feuillage en avant-plan qui encadrera tout le reste du cédérom, comme le rappel omniprésent d'une aventure épique en jungle tropicale? Le titre le mentionne, la jaquette le souligne, il s'agit d'un voyage. "Angkor, le terme résonne comme une invitation au voyage". Un nom évocateur, d'aventure, de jungle et de temples oubliés. Un peu comme Pondichéry ou Chandernagor, mais avec la jungle et les temples en plus ? Voyageons, donc.

Autour de l' histoire de ce touriste, "la découverte du voyageur ", filent deux histoires parallèles: la civilisation angkorienne présentée par Jayavarman VII et une compilation choisie de légendes contées par une danseuse du ballet royal, Sophéa, aimée jadis (mais en vain) de son grandpère. Point commun de ces trois histoires: un seul et même itinéraire d'une semaine, chaque journée offrant un nombre inégal de sites, de sujets ou de 
légendes. Sous la conduite de leur guide, chaque histoire est un cheminement illustré d'ambiances sonores, de textes, de photos et de séquences vidéo. Entre ces trois parcours linéaires qui se dévident automatiquement (on peut néanmoins sauter les étapes), quelques éphémères passerelles interactives ont été jetées à partir de la visite centrale pour s'évader d'un récit vers l'un des deux autres ou vers un glossaire et des cartes (dont certaines présentent quelques perspectives malheureusement incohérentes). Peu de chance de se perdre dans cette trame classique, mais un programme général est cependant offert avec le planning des interventions de chaque orateur. $\mathrm{Ni}$ bibliographie, ni sources, les crédits officiels ne permettent aucune ouverture hors de ce cédérom.

Suivons d'abord le récit central, la découverte du "voyageur". Ambiances sonores, commentaires et petites images vidéo se succèdent ou s'additionnent tels des timbres sur un fond - principalement - noir. Premier constat, gênant, même - et surtout - sur grand écran: l'avarice de l'illustration. Ce problème ne se limite malheureusement pas seulement au début du récit puisqu'on le retrouvera constamment. Les images ne sont certes pas si rares, mais la priorité donnée aux séquences vidéo semble s'être répercutée directement dans leur format très réduit, les rendant peu attractives. Ce regret vaudra aussi pour les deux autres récits dont la mise en page comporte moins de séquences vidéo (ils se rapprochent plus d'un diaporama), mais où l'illustration restera toujours limitée. Suivons cependant le "voyageur " dans un itinéraire digne de Mouhot ou de Malraux, à mi-chemin entre rêve et réalité, entre voyage vécu et voyage imaginé, sans grande considération pour la géographie élémentaire des environs (remonter en barque la rivière en aval de Siemreap pour arriver à Bakong). Peu avare de commentaires oniriques et d'impressions colorées, le voyageur est le seul à évoluer dans des images en couleur, Jayavarman vII et Sophéa restant cantonnés dans les sépias et les pastels, onirisme oblige. Sur la trace aventureuse de son ancêtre, le voyageur "doit" dormir dans les pagodes ou dans les temples. On attendait alors de la séquence de "Rêve au Bayon", l'onirisme étant à l'honneur, qu'elle rende un peu de cette magie du lieu un soir de pleine lune. Malheureusement on pourra rester encore sceptique devant un écran qui ne montre guère plus que quelques effets de nuit américaine sur les visages du Bayon, avec en fond le chant des grillons sans les moustiques. Ce "rêve" est au moins révélateur, sans avoir à réviser Freud auparavant, de la pauvreté de l'iconographie mise en œuvre pour nous transporter à Angkor.

Passons alors côté Histoire, avec un grand $\mathrm{H}$ et un grand Jayavarman VII qui, au terme d'une vie que les archéologues ont allègrement remplie depuis Stern et Codès, revoit avec une certaine nostalgie l'ensemble de l'histoire angkorienne en sépia. Son récit mêle l'histoire des historiens et de beaux passages d'inscriptions, tout en empruntant bien sûr à l'inévitable Zhou Daguan. Les premiers jours présentent un tableau cohérent et imagé de la 
chronologie angkorienne en insistant sur les phases clefs et spectaculaires. Certains détails témoignent de la contribution de la "validation scientifique", en particulier la patte de Claude Jacques (la date de 880 AD pour Prah Kô, ou la triade d'Umâgangâpatîçvara revisitée en Vishnu - Lakshmi - Bhûmi). Mais cette contribution se retrouve souvent diluée sous la "réécriture" substantielle du réalisateur $\mathrm{J}$. Frydman et les effets de style se font alors plus fréquents: "Moi, Jayavarman VII...", "Demain, je partirai...". "Jayavarman VII, ce héros au sourire si doux..." nous a cependant été épargné alors que les statues-portraits de Jayavarman VII s'y prêtaient bien. L'environnement sonore renforce encore ces effets avec des intonations de péplum hollywoodien: après la bataille contre les Chams, on s'attendrait à voir surgir d'Angkor Thom Liz Taylor (dans le rôle de Jayarâjadevi) au bras de Richard Burton. D'ailleurs, à partir d'Angkor Thom, le style - sans Liz - prend le pas sur l'histoire qui y perd sa majuscule, au bénéfice, certes, de l'évocation de la cité de Jayavarman VII. Notons qu'en achevant cette histoire avec les vieux jours du célèbre souverain, la période de l'après Jayavarman VII, pourtant chère à Claude Jacques, est niée, accréditant ainsi le mythe d'une Angkor abandonnée à la voracité végétale.

Suivons enfin dans un cadre rosâtre le "pèlerinage" de la danseuse Sophéa alors que plane le souvenir vague mais tenace de Saramani, sa consœur louée par Charles Meyer (toujours pas cité). Sophéa parle cambodgien, au moins les premières secondes de chaque séquence, avant qu'une traductrice ne la couvre. Ce détail est assez rafraîchissant après avoir écouté Jayavarman VII s'exprimer dans un français ténébreux. Mais la touche d'authenticité dure à peine assez pour donner vie aux quelques récits sans ordre apparent que nous contera cette danseuse. Récits peu nombreux car, au hasard d'un détour par le planning général, on découvre qu'elle est nettement sous employée avec seulement 18 sujets contre 24 pour Jayavarman VII et 26 pour le voyageur.

Un dernier point commun entre ces trois récits, le septième jour: Angkor Vat. Les contorsions géographiques de notre "voyageur", l'étonnement de voir un grand souverain bouddhiste choisir comme dernier pèlerinage un temple vishnuite, les déambulations de Sophéa, tout cela avait donc pour but de ménager le suspens jusqu'à l'apothéose de la danse d'une Apsara à Angkor Vat. Final stéréotypé, digne des brochures de tour opérateur, le spectacle reste encore imaginaire avec son iconographie liliputienne.

Trois histoires donc, qui se déroulent sans nœuds mais sans atteindre une quelconque troisième dimension. Après trois heures en spectateur sans grand spectacle, après avoir abandonné tout intérêt pour d'éventuels zapping, nous attendions tout de même plus de ce "documentaire multimédia" au ton résolument onirique: ces déambulations monotones sur un itinéraire à la logique douteuse et les récits stylisés de nos trois guides laissent un amer goût de vide. Et le voyage promis, notre voyage? Il était pourtant mis 
à l'honneur en sous-titre et sur la jaquette avec cette citation de Victor Ségalen: "Le voyage, confrontation sur le terrain du réel et de l'imaginaire". Cette citation était-elle, après tout, bien adaptée à ce voyage virtuel sur cédérom qui, au final, est accaparé par l'imaginaire du réalisateur-scénariste Julien Frydman et par ses souvenirs de repérages? Le "terrain du réel" reste absent et l'aspect didactique particulièrement restreint, à l'opposé de certaines publications de Claude Jacques ou de Thierry Zéphir. Ce cédérom pourra-t-il réveiller l'imaginaire angkorien au détour de vos prochains rêves... et alors motiver inconsciemment vos prochaines vacances? Un documentaire télévisé (libéré des 650Mo et certainement plus rapide) aurait eu vraisemblablement au moins le même résultat.

\section{Angkor, 10 siècles de fascination}

Ce second cédérom s'ouvre sur un long diaporama plein écran qui mérite à lui seul de suivre un conseil du "Lisez-moi" préalable: configurer l'écran en un millier de couleurs. Classique, bien qu'inventif, l'écran y décline de superbes photos de Bruno Thomassin, mariant monuments, ambiances et habitants. Mais, trêve de belles images, quitte à y revenir plus tard, un clic présente le sommaire principal. Celui-ci, dont le soin graphique se retrouvera constamment plus tard, jusque dans la signalétique la plus banale, propose sept thèmes qui sont autant de facettes d'Angkor et de pistes pour découvrir ce cédérom: quatre "voyages dans le temps" et trois "chefs-d'œuvre du génie khmer". Par ailleurs, un menu disponible à tout moment permet des accès directs aux "ressources encyclopédiques" (glossaire, atlas et histoire) et aux crédits... Un jeu de piste s'annonce donc en perspective car, chaque piste pouvant mener à d'autres embranchements secondaires, le parcours tourne parfois au labyrinthe. L'ensemble apparaît comme une large arborescence à plusieurs niveaux qui reste essentiellement accessible depuis le tronc du sommaire, bien que quelques parcours relient transversalement des thèmes liés aux temples. Le sommaire servira donc aussi avantageusement de point de repère si, d'aventure, un cul-de-sac apparaît au terme d'une déambulation.

Pas de justification donc à ce cédérom, autre que celle d'aider à découvrir, à travers lui, des facettes d'Angkor. Ici aussi, le ton est déjà donné: une iconographie riche et de qualité, une vaste palette didactique de thèmes complémentaires et une liberté de choix "interactifs". Euphémisme, cette dernière implique la participation active de l'utilisateur. Visiter Angkor, c'est pour certains, jouir de l'effort (le sien et celui des autres), de l'ambiance et parfois de la lecture du Guide Glaize. Transposition virtuelle, visiter ce cédérom exigera le mouvement (parfois quasiment physique tellement le clic est roi) dans des itinéraires copieusement illustrés et patiemment expliqués. Cliquons donc (pour les utilisateurs de trackpad, il peut être conseillé de brancher une souris). 
Au hasard, commençons par "la création du monde". Le panthéon brahmanique et la cosmologie hindoue y sont intelligemment expliqués et illustrés par une progression de plusieurs séquences. Le travail informatique des images apparaît déjà dans l'aménagement de nombreux effets, en particulier dans l'animation très visuelle du célèbre relief du barattage d'Angkor Vat. Les résultats de ces effets sont pourtant inégaux, en particulier lors des séquences en Quick Time VR (QTVR) où la "réalité virtuelle" peu spectaculaire d'un objet en deux dimensions se fait au prix de la qualité de l'image.

"La cité hydraulique" présente fidèlement les thèmes principaux de l'article du même nom de B.P. Groslier, complétés par les dernières recherches de Jacques Dumarçay sur ce domaine. Régimes et ouvrages hydrauliques, temples, capitales, canaux, zones irriguées... l'ensemble se succède et/ou se superpose clairement, renforçant l'image d'un palimpseste, basé sur une image Spot en vraies couleurs. L'effet d'ensemble est réussi, autant que la théorie elle-même qui, on peut le noter sans devoir au préalable jeter "un pavé dans le baray", n'a pas que des adeptes. D'ailleurs, cette simplification volontaire met aussi en valeur les zones encore un peu sombres de ce schéma (d'un point de vue graphique, l'alimentation du baray oriental est tellement tentante depuis la rivière de Roluos... qu'elle apparaît subrepticement alors que le commentaire ne parle que de celle de Siemreap; malheureusement, elle disparaît quelques secondes plus tard !). Notons enfin que quelques détails hâtifs se sont glissés dans la restitution infographique (par exemple, les canaux fantaisistes de Haryharâlaya, pourtant bien visibles sur la photographie aérienne qui leur sert de fond).

Sous le singulier de "Mémoire d'explorateurs" sont évoquées les réactions et interventions des étrangers qui se sont succédés à Angkor, de Zhou Daguan à B.P. Groslier, englobant ainsi les phases principales de la découverte d'Angkor, de la naissance du mythe et des travaux entrepris par l'EFEO depuis le début du siècle. Peut-on noter que cette approche large a - entre autres - l'avantage de traiter de la période coloniale sans angle nostalgique en la replaçant dans son contexte? On remarquera bien évidemment dans cette approche l'influence de La forêt de pierre de Bruno Dagens (voir la bibliothèque du cédérom). Plusieurs modifications par rapport à cet ouvrage justifient néanmoins de s'engager dans cette piste du cédérom: le nouveau support médiatique apporte ambiances sonores, animations (nous sommes toujours en pleine interactivité, à vous donc de cliquer pour que le crocodile dévore les singes) et une iconographie amplement revisitée tirant profit du grand format (les photos de Thomson par exemple).

Considérée comme le quatrième "voyage dans le temps", le temps présent, "La vie à l'ombre des temples" repose un peu de l'interactivité effrénée de la séquence précédente et propose des récits fluides sur les éléments structurants de la vie rurale (les saisons, le lac, le riz, le village, la pagode...). Les 
lumineuses photographies de Bruno Thomassin et les commentaires tirés de la pratique de Fabienne Luco rendent clairement communicative la séduction qu'exerce le monde rural contemporain aux portes d'Angkor. Simple (hormis quelques animations QTVR égarées), mais efficace.

Reposés? Souris neuve? Abordons alors le premier des trois "chefsd'œuvre du génie khmer", "les temples d'Angkor". Trois sous-aspects sont proposés. Le premier constitue un dossier de fiches individuelles où chaque temple présente une description de quelques lignes (la date de Prah Kô est ici de $879 \mathrm{AD}$ ), une modélisation (plan, façade et perspective), quelques photos (deux ou trois, une dizaine à Angkor Vat, certes, toujours aussi réussies, mais on s'attendait parfois à plus) et en option, un survol en vidéo (de basse définition, il fait double emploi avec la modélisation) ou des panoramiques QTVR. Ces derniers, dont la définition médiocre est évidente lors des zoom, sont judicieusement implantés et apportent, avec toutes les limites du procédé, une certaine matérialité aux espaces. Remarquons aussi les modélisations schématiques qui supportent assez bien le double-clic pour les observer en plein écran (malgré quelques rares incohérences, comme cette différence injustifiée de forme de couverture entre les bibliothèques de Bakong et celle de Prah Kô). Elles soulignent divers traits majeurs de l'architecture khmère: un vocabulaire volumétrique réduit, des principes de composition strictement géométriques et une grande diversité des configurations obtenues. Un second aspect nous entraîne vers trois temples (Bakheng, Neak Pean et Bayon) où symbolisme et effets perspectifs sont démasqués à l'aide de nouvelles modélisations et de séquences animées interactives dont certaines révèlent surtout, non sans quelque ironie, les potentialités de tricherie de l'outil informatique. Ne pas rater dans les environs un passage mémorable d'une interview de B.P. Groslier en 1966. Le troisième aspect concerne enfin l'ornementation qui recouvre les architectures angkoriennes. "Décorez Ta Kev" pourrait être le sujet de cette séquence/exercice particulièrement didactique, illustrée de photos et de commentaires raisonnablement courts. Cet essai d'habillage permet d'introduire indirectement quelques éléments de terminologie architecturale.

Après les temples, "la statuaire khmère". C'est l'objet du thème suivant qui présente, encore, trois choix: la population divine via l'accès à une série de fiches d'identité illustrées de nombreuses photographies de statues khmères, un cours de décodage des techniques narratives en cuvre sur quelques exemples choisis de linteaux, frontons et bas-reliefs, et une visite virtuelle du Musée National de Phnom Penh. Notons que le second point reprend une technique de décomposition, déjà rencontrée lors de la création du monde, qui exprime clairement le contraste entre le fouillis apparent de certaines scènes et la limpidité de la démonstration que nous prodigue Natalia Rodriguez. Regrettons juste qu'ici encore, il faille tant cliquer un peu partout pour ne pas arriver dernier de la classe. Après ceci, la pratique d'une déambulation en 
QTVR au Musée sonne comme un clin d'oeil à l'exposition du Grand Palais et une récréation (pas pour la souris) où tout est permis, même, sacrilège du virtuel, de manier certaines pièces. Cet intermède phnom-penhois est sans conteste la plus justifiée des séquences QTVR.

Dernier thème du sommaire, "Légendes et traditions" offre trois diaporamas (plus ou moins interactifs) illustrant une version de la légende du Roi Lépreux, le Reamker et la danse traditionnelle. Ici encore, le traitement et l'organisation des images sont mis au service d'un récit pédagogique, fermement basé sur une iconographie remarquablement choisie. Notons par exemple une intéressante couverture inédite des peintures du Reamker d'un Vat de Siemreap (due à Olivier de Bernon, FEMC), ou la gestuelle de la multitude d" Apsaras" d'Angkor Vat qui, patiemment détourées, acquièrent une troublante réalité.

Après avoir parcouru tous les choix du sommaire, quitter serait déjà bien mérité. S'il vous reste encore une souris et un peu de curiosité pour vous aventurer dans le menu inférieur, ces quelques annexes vous entraînent vers de nouvelles pistes, certes plus "encyclopédiques", mais toujours aussi didactiques. Avec une présentation particulièrement sérieuse et neutre par rapport à ce que nous avons pu voir précédemment, l'atlas joue des échelles et de la superposition des éléments forts de l'environnement (reliefs, hydrologie...) pour offrir quelque 25 cartes différentes. Outre de très rares coquilles (Preah Vihear, au Nord-Est de Pimay, frise l'incident diplomatique), la surprise réside dans l'interactivité qui y est particulièrement limitée: on ne peut pas, par exemple, accéder aux temples depuis leur carte. Oubli, ou ironie particulière des concepteurs pour refuser la réaction primaire du novice en interactivité ? "Histoire" correspond plutôt à un atlas historique qui offre un récapitulatif des phases principales, illustré de rares photographies (inégales mais parfois savoureuses), de textes et de commentaires (visez l'oreille). Notons que le monde ne s'y arrête pas à Angkor et qu'il continue jusqu'au présent, ou du moins jusqu'à 1953 et donc sans évoquer les années 70. La bibliothèque, enfin, ouvre le cédérom à la manière d'un "Pour en savoir plus" et rend hommage aux ouvrages et aux travaux qui fournirent la matière de ce cédérom. Parmi ceux-ci, notons que, contrairement aux autres références de la bibliographie, seul le célèbre article de B.P. Groslier, pourtant deux fois cité et largement exploité, ne porte aucune indication de provenance ou de date... tellement cela devait sembler évident. Remarquons pour finir quelques belles couvertures, dont une publication de l'EFEO retapissée en lettres d'or à Bangkok par White Lotus.

L'ordinateur éteint pour refroidir le silicium, on peut se surprendre à contempler naïvement ce cédérom de 650 Mo en se demandant où a pu être stocké tout ce que l'on vient de voir. Encore de la place ? Peut-être un peu: un compte rendu paru au début 1997 (Dossier d'Archéologie, n²21, p.79) assurait avant même que le cédérom soit achevé, que nous devions y trouver 
en plus une "visite guidée des chantiers de restauration actuellement en cours, etc.". Nous ne pourrons donc pas jouer à "Anastylosez votre temple" ou à "Composez votre équipe internationale d'experts". Les comptes rendus, même préfabriqués, sont toutefois utiles: celui-ci montre aussi que l'option consistant à louer des guides célèbres avait été envisagée: Indra, Jayavarman VII, l'explorateur occidental (Mouhot?) et "l'enfant khmer". On ne saurait regretter l'abandon de ce casting éloquent, la narration y gagnant en objectivité et en dépouillement. De même, on reconnaîtra que le cédérom n'y a perdu ni en logique, ni en ambiance, la densité, la qualité et le choix de l'iconographie se chargeant allègrement "d'émouvoir et d'éveiller la curiosité". Au final, le caractère didactique est omniprésent mais se laisse oublier sous l'environnement et les cheminements proposés qui forment autant de niveaux de lecture allant de la recherche de connaissances à la simple immersion visuelle. Les heures passées à cliquer (de 3 à 6) se retrouvent ainsi valorisées par le kaléidoscope d'images et par la densité d'informations disponibles sous les formes les plus diverses. Marier la forme et le fond ne fut pas le dernier soucis du réalisateur Fabrice Cerezales et de ses collaborateurs pour ce cédérom qui ne cache ni ses sources ni l'environnement autour duquel il a été conçu, à Angkor même.

\section{Épilogue}

Après quelques heures passées avec ces deux cédéroms, il est difficile de ne pas s'interroger sur la fonction de ce type de support, et donc sur son contenu. Le premier cédérom, Angkor, Cité royale [Récit de voyages], apparaît comme une œuvre personnelle, la vision d'un réalisateur favorisant l'évocation du mythe angkorien au dépens de la réalité de l'objet. Au vu du résultat des récits linéaires et des choix iconographiques, on peut alors raisonnablement douter qu'un cédérom soit le support adéquat pour ce qui ressemble plutôt à un montage vidéo, à peine ponctué par quelques diaporamas à l'interactivité limitée. Peut-être les nouvelles générations de cédéroms (DVD) permettrontelles dans un avenir proche de dépasser cette inaptitude. Le second, Angkor, 10 siècles de fascination, s'apparente à une synthèse didactique, à un manuel richement illustré sous un environnement informatique interactif. Le scénario est alors laissé au libre choix de l'utilisateur - au moins jusqu'à un certain niveau - le réalisateur tablant sur la convivialité de l'environnement, parfois ludique, pour transmettre des connaissances de base sur un sujet complexe. Nul doute donc que ce choix soit plus adapté aux amateurs de dilettante informatique néanmoins soucieux de ne pas y perdre totalement leur temps. Ce cédérom leur propose alors un ouvrage de vulgarisation, au sens noble, sur un nouveau médium.

Dans le contexte de l'exposition d'Art Khmer qui, on s'en doute, joua pour beaucoup dans la genèse de ces deux cédéroms, d'autres choix auraient encore été possibles dont ces deux, caricaturaux: une promotion pure et 
simple d'Angkor en tant que destination touristique, avec numéros de téléphone et agences de voyages (à l'image du cédérom trilingue "Saint Denis 98" sorti dans la foulée du Stade et de la Coupe du Monde) ou bien la mise à disposition d'une somme de ressources documentaires "brutes" sur Angkor (comme le récent "Bulletin des Amis du Vieux Huê")... Ces deux positions extrêmes pourraient correspondre aux prolongements logiques de nos deux cédéroms sur Angkor, et montrer aussi leurs limites. Pour le premier, une invitation au voyage "gratuite", mettant en avant le nom d'Angkor à travers un style et quelques clichés séducteurs, mais sans donner finalement les bases du voyage ou même les "adresses utiles". Le second, ouvrant sur une large palette séduisante d'informations d'origine scientifique sur la richesse d'Angkor, mais restreint cependant à un aperçu général. Ni guide pratique, ni ouvrage scientifique, ces deux cédéroms risquent d'être aussi éphémères que l'exposition qui a motivé leur réalisation, et de passer beaucoup moins d'hivers que le catalogue de l'exposition, qu'un "Guide Glaize", qu'un "Pèlerin d'Angkor" ou encore qu'une "Forêt de pierre"!

Christophe Pottier

1. Les Monuments du Groupe d'Angkor, Guide par Maurice Glaize, J. Maisonneuve, $3^{\mathfrak{e}}$ édition, Paris, 1963; Un pèlerin d'Angkor par Pierre Loti, Calmann Lévy, Paris 1912; Angkor, la forêt de pierre par Bruno Dagens, collection "Découvertes", Gallimard, Paris, 1989. 